\title{
Affine Nil-Hecke Algebras and Braided Differential Structure on Affine Weyl Groups
}

\author{
by
}

Anatol N. Kirillov and Toshiaki Maeno

\begin{abstract}
We construct a model of the affine nil-Hecke algebra as a subalgebra of the NicholsWoronowicz algebra associated to a Yetter-Drinfeld module over the affine Weyl group. We also discuss the Peterson isomorphism between the homology of the affine Grassmannian and the small quantum cohomology ring of the flag variety in terms of the braided differential calculus.
\end{abstract}

2010 Mathematics Subject Classification: Primary 16T30; Secondary 20F55, 14 M17.

Keywords: nil-Hecke algebra, Hopf algebra, affine Grassmannian.

\section{Introduction}

The cohomology ring of the flag variety is a fundamental object of research in the study of the Schubert calculus. Fomin and the first author [4] gave a combinatorial model of the cohomology ring $H^{*}\left(F l_{n}\right)$ of the flag variety of type $A$ as a commutative subalgebra of a quadratic algebra $\mathcal{E}_{n}$. It is remarkable that the algebra $\mathcal{E}_{n}$ has a natural quantum deformation $\mathcal{E}_{n}^{q}$ so that $\mathcal{E}_{n}^{q}$ contains the quantum cohomology ring $Q H^{*}\left(F l_{n}\right)$ as a commutative subalgebra.

It has been observed by Milinski and Schneider [12] and by Majid [11] that the defining relations of the Fomin-Kirillov quadratic algebra $\mathcal{E}_{n}$ are understandable from the viewpoint of a certain kind of braided Hopf algebra called the NicholsWoronowicz algebra. Bazlov [2] constructed a model of the coinvariant algebra of the finite Coxeter groups as a commutative subalgebra of the Nichols-Woronowicz

Communicated by M. Kashiwara. Received September 13, 2010. Revised August 31, 2011.

A. N. Kirillov: Research Institute for Mathematical Sciences, Kyoto University,

Sakyo-ku, Kyoto 606-8502, Japan;

e-mail: kirillov@kurims.kyoto-u.ac.jp

T. Maeno: Department of Electrical Engineering, Kyoto University,

Sakyo-ku, Kyoto 606-8501, Japan;

e-mail: maeno@kuee.kyoto-u.ac.jp 
algebra. At the same time, the nil-Coxeter algebra, which is dual to the coinvariant algebra, is also realized as a subalgebra of the Nichols-Woronowicz algebra.

The braided analogue of the exterior algebra was introduced by Woronowicz [15] for the study of differential forms on quantum groups. For a given braided vector space $M$ over a field $K$ of characteristic zero, the braided analogue $\mathcal{B}(M)$ of the symmetric algebra of $M$ is similarly defined to be the quotient of the free tensor algebra of $M$ by the kernel of the braided symmetrizer. It is known that the algebra $\mathcal{B}(M)$ is a braided graded Hopf algebra characterized by the following conditions:

(1) $\mathcal{B}^{0}(M)=K$,

(2) $\mathcal{B}^{1}(M)=M=\{$ primitive elements in $\mathcal{B}(M)\}$,

(3) $\mathcal{B}^{1}(M)$ generates $\mathcal{B}(M)$ as an algebra.

The algebra is related to earlier ideas in [13]. The Hopf algebra generated by the primitive elements has been studied by Nichols [13] and named the Nichols algebra by Andruskiewitsch and Schneider [1]. The actual braided Hopf algebra structure of $\mathcal{B}(M)$ has its origins in the work of Majid, notably [9], [10]. The tensor algebras $T M$ and $T M^{*}$ are primitively generated braided Hopf algebras [9] and are dually paired as braided Hopf algebras by extending the pairing in degree 1 . This pairing is typically degenerate and the quotient by the kernel on each side yields braided Hopf algebras $\mathcal{B}(M), \mathcal{B}\left(M^{*}\right)$ which are now nondegenerately paired and canonically associated to $M$. Majid used this construction in [10]. In this paper we will call $\mathcal{B}(M)$ the Nichols-Woronowicz algebra following [2].

The aim of this paper is to construct the nil-Hecke algebra as a subalgebra of an extension of the Nichols-Woronowicz algebra $\mathcal{B}_{\text {aff }}$ associated to a YetterDrinfeld module over the affine Weyl groups. Our construction is analogous to the one in $[2$, Section 6$]$.

It is known that the affine Grassmannian $\widehat{\mathrm{Gr}}:=G(\mathbb{C}((t))) / G(\mathbb{C}[[t]])$ of a semisimple Lie group $G$ is homotopic to the loop group $\Omega K$ of the maximal compact subgroup $K \subset G$. The homology $H_{*}(\widehat{\mathrm{Gr}}) \cong H_{*}(\Omega K)$ carries an associative algebra structure induced by the Pontryagin product. The strucuture of the Pontryagin ring $H_{*}(\Omega K)$ has been determined by Bott [3]. The Schubert calculus for Kac-Moody flag varieties was studied by Kostant and Kumar [6] by using the nilHecke algebra. Peterson [14] stated that the torus-equivariant homology $H_{*}^{T}(\widehat{\mathrm{Gr}})$ of the affine Grassmannian is isomorphic to the so-called Peterson subalgebra of the affine nil-Hecke algebra. So our construction gives a model of $H_{*}^{T}(\widehat{\mathrm{Gr}})$ as a commutative subalgebra of the Nichols-Woronowicz algebra $\mathcal{B}_{\text {aff }}(S)$ (see Theorem 3.4). 
Peterson [14] also pointed out that the Pontryagin ring $H_{*}^{T}(\widehat{\mathrm{Gr}})$ is isomorphic to the small quantum cohomology $\operatorname{ring} Q H_{T}^{*}(G / B)$ of the corresponding flag variety $G / B$ as an algebra after a suitable localization. The affine Bruhat operator acting on $H_{*}^{T}(\widehat{\mathrm{Gr}})$ introduced by Lam and Shimozono [7] gives an explicit comparison between the multiplicative structure of $H_{*}^{T}(\widehat{\mathrm{Gr}})$ and that of $Q H_{T}^{*}(G / B)$. In this paper, we will realize the affine Bruhat operator as a braided differential operator (see Section 3 for details) acting on our algebra $\mathcal{B}_{\text {aff }}$.

\section{§1. Affine nil-Hecke algebra}

Let $G$ be a simply-connected semisimple complex Lie group and $W$ its Weyl group. Denote by $\Delta$ the set of roots. We fix the set $\Delta_{+}$of positive roots by choosing a set of simple roots $\alpha_{1}, \ldots, \alpha_{r}$. The Weyl group $W$ acts on the weight lattice $P$ and the coroot lattice $Q^{\vee}$ of $G$. The affine Weyl group $W_{\text {aff }}$ is generated by the affine reflections $s_{\alpha, k}, \alpha \in \Delta, k \in \mathbb{Z}$, with respect to the affine hyperplanes $H_{\alpha, k}:=$ $\{\lambda \in P \otimes \mathbb{R} \mid\langle\lambda, \alpha\rangle=k\}$. The affine Weyl group is the semidirect product of $W$ and $Q^{\vee}$, i.e., $W_{\text {aff }}=W \ltimes Q^{\vee}$. The affine Weyl group $W_{\text {aff }}$ is generated by the simple reflections $s_{1}:=s_{\alpha_{1}, 0}, \ldots, s_{r}:=s_{\alpha_{r}, 0}$ and $s_{0}:=s_{\theta, 1}$ where $\theta=-\alpha_{0}$ is the highest root. The affine Weyl group $W$ has the presentation as a Coxeter group as follows:

$$
W_{\text {aff }}=\left\langle s_{0}, \ldots, s_{r} \mid s_{0}^{2}=\cdots=s_{r}^{2}=1,\left(s_{i} s_{j}\right)^{m_{i j}}=1\right\rangle .
$$

Definition 1.1. The affine nil-Coxeter algebra $\mathbb{A}_{0}$ is the associative $\mathbb{Q}$-algebra generated by $\tau_{0}, \ldots, \tau_{r}$ subject to the relations

$$
\tau_{0}^{2}=\cdots=\tau_{r}^{2}=0, \quad\left(\tau_{i} \tau_{j}\right)^{\left[m_{i j} / 2\right]} \tau_{i}^{\nu_{i j}}=\left(\tau_{j} \tau_{i}\right)^{\left[m_{i j} / 2\right]} \tau_{j}^{\nu_{i j}},
$$

where $\nu_{i j}:=m_{i j}-2\left[m_{i j} / 2\right]$.

For a reduced expression $x=s_{i_{1}} \cdots s_{i_{l}}$ of an element $x \in W_{\text {aff }}$, the element $\tau_{x}:=\tau_{i_{1}} \cdots \tau_{i_{l}} \in \mathbb{A}_{0}$ is independent of the choice of the reduced expression of $x$. It is known that $\left\{\tau_{x}\right\}_{x \in W_{\text {aff }}}$ form a linear basis of $\mathbb{A}_{0}$.

The nil-Coxeter algebra $\mathbb{A}_{0}$ acts on $S:=\operatorname{Sym} P_{\mathbb{Q}}$ via

$$
\begin{aligned}
\tau_{0}(f) & :=\partial_{\alpha_{0}}(f)=-\left(f-s_{\theta, 0} f\right) / \theta, \\
\tau_{i}(f) & :=\partial_{\alpha_{i}}(f)=\left(f-s_{\alpha_{i}, 0} f\right) / \alpha_{i}, \quad i=1, \ldots, r,
\end{aligned}
$$

for $f \in S$.

Definition $1.2([6])$. The nil-Hecke algebra $\mathbb{A}$ is defined to be the cross product $\mathbb{A}_{0} \ltimes S$, where the cross relation is given by

$$
\tau_{i} f=\partial_{\alpha_{i}}(f)+s_{i}(f) \tau_{i}, \quad f \in S, i=1, \ldots, r .
$$


Here, we summarize some known results on the homology of the affine Grassmannian. The affine Grassmannian $\widehat{\mathrm{Gr}}:=G(\mathbb{C}((t))) / G(\mathbb{C}[[t]])$ is homotopic to the loop group $\Omega K$ of the maximal compact subgroup $K \subset G$. Let $T \subset G$ be the maximal torus. We consider the $T$-equivariant homology group $H_{*}^{T}(\widehat{\mathrm{Gr}})$ over $\mathbb{Q}$. An associative algebra structure on $H_{*}^{T}(\widehat{\mathrm{Gr}}) \cong H_{*}^{T}(\Omega K)$ is induced from the group multiplication

$$
\Omega K \times \Omega K \rightarrow \Omega K
$$

It is known that the algebra $H_{*}^{T}(\widehat{\mathrm{Gr}})$ is commutative. The algebra $H_{*}^{T}(\Omega K)$ is called the Pontryagin ring.

We regard the $T$-equivariant homology $H_{*}^{T}(\widehat{\mathrm{Gr}})$ as an $S$-algebra by identifying $S=H_{T}^{*}(p t)$. The diagonal embedding

$$
\Omega K \rightarrow \Omega K \times \Omega K
$$

induces a coproduct on $H_{*}^{T}(\widehat{\mathrm{Gr}})$. Let $J \subset \mathbb{A}$ be the left ideal of $\mathbb{A}$ generated by the elements $\tau_{w}, w \in W \backslash\{\mathrm{id}\}$. The centralizer $Z_{\mathbb{A}}(S)$ of $S$ in $\mathbb{A}$ is called the Peterson subalgebra of $\mathbb{A}$.

Proposition 1.1 ([14]). Let $\left\{\xi_{x} \mid x \in W_{\text {aff }}\right\}$ be the Schubert basis of the Tequivariant homology $H_{*}^{T}(\widehat{\mathrm{Gr}})$. The T-equivariant homology $H_{*}^{T}(\widehat{\mathrm{Gr}})$ is naturally identified with the Peterson subalgebra $Z_{\mathbb{A}}(S)$ via the $S$-algebra isomorphism $j$ : $H_{*}^{T}(\widehat{\mathrm{Gr}}) \rightarrow Z_{\mathbb{A}}(S)$ characterized by the following conditions:

(1) $j\left(\xi_{x}\right)=\tau_{x} \bmod J$ for $x \in W_{\text {aff }}$,

(2) $j(\xi) \xi^{\prime}=\xi \xi^{\prime}$ for $\xi, \xi^{\prime} \in H_{*}^{T}(\widehat{\mathrm{Gr}})$.

\section{$\S 2$. Nichols-Woronowicz algebra for affine Weyl groups}

We briefly recall the construction of the Nichols-Woronowicz algebra associated to a braided vector space. Let $M$ be a vector space over a field of characteristic zero and $\psi: M^{\otimes 2} \rightarrow M^{\otimes 2}$ be a fixed linear endomorphism satisfying the braid relations $\psi_{i} \psi_{i+1} \psi_{i}=\psi_{i+1} \psi_{i} \psi_{i+1}$ where $\psi_{i}: M^{\otimes n} \rightarrow M^{\otimes n}$ is a linear endomorphism obtained by applying $\psi$ to the $i$-th and $(i+1)$-st components. Denote by $s_{i}$ the simple transposition $(i, i+1) \in S_{n}$. For any reduced expression $w=s_{i_{1}} \cdots s_{i_{l}} \in S_{n}$, the endomorphism $\Psi_{w}=\psi_{i_{1}} \cdots \psi_{i_{l}}: M^{\otimes n} \rightarrow M^{\otimes n}$ is well-defined. The Woronowicz symmetrizer (cf. [15]) is given by $\sigma_{n}:=\sum_{w \in S_{n}} \Psi_{w}$. The operator $\sigma_{n}$ is also called the braided integer in [9]. 
Definition 2.1 (cf. [15]). The Nichols-Woronowicz algebra associated to a braided vector space $M$ is defined by

$$
\mathcal{B}(M):=\bigoplus_{n \geq 0} M^{\otimes n} / \operatorname{Ker}\left(\sigma_{n}\right),
$$

where $\sigma_{n}: M^{\otimes n} \rightarrow M^{\otimes n}$ is the Woronowicz symmetrizer.

Definition 2.2. A vector space $M$ is called a Yetter-Drinfeld module over a group $\Gamma$ if the following conditions are satisfied:

(1) $M$ is a $\Gamma$-module,

(2) $M$ is $\Gamma$-graded, i.e. $M=\bigoplus_{g \in \Gamma} M_{g}$, where $M_{g}$ is a linear subspace of $M$,

(3) for $h \in \Gamma$ and $v \in M_{g}, h(v) \in M_{h g h^{-1}}$.

The Yetter-Drinfeld module $M$ over a group $\Gamma$ is naturally braided with the braiding $\psi: M^{\otimes 2} \rightarrow M^{\otimes 2}$ defined by $\psi(a \otimes b)=g(b) \otimes a$ for $a \in M_{g}$ and $b \in M$.

In the following we are interested in the Yetter-Drinfeld module over the affine Weyl group $W_{\text {aff }}$. Denote by $t_{\lambda} \in W_{\text {aff }}$ the translation by $\lambda \in Q^{\vee}$. We define a Yetter-Drinfeld module $V_{\text {aff }}$ over $W_{\text {aff }}$ by

$$
V_{\mathrm{aff}}:=\bigoplus_{\alpha \in \Delta, k \in \mathbb{Z}} \mathbb{Q} \cdot[\alpha, k] /([\alpha, k]+[-\alpha,-k]),
$$

where $W_{\text {aff }}$ acts on $V_{\text {aff }}$ by

$$
w[\alpha, k]:=[w(\alpha), k], \quad w \in W, \quad t_{\lambda}[\alpha, k]:=[\alpha, k+(\alpha, \lambda)], \quad \lambda \in Q^{\vee} .
$$

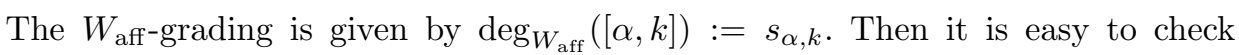
the conditions in Definition 2.1. Now we have the Nichols-Woronowicz algebra $\mathcal{B}_{\text {aff }}:=\mathcal{B}\left(V_{\text {aff }}\right)$ associated to the Yetter-Drinfeld module $V_{\text {aff }}$.

Let $\mathcal{B}_{W}$ be the Nichols-Woronowicz algebra associated to the Yetter-Drinfeld module $V=\bigoplus_{\alpha \in \Delta} \mathbb{Q} \cdot[\alpha] /([\alpha]+[-\alpha])$ as in [2, Section 4].

Lemma 2.3. (1) We have a surjective homomorphism $\pi: \mathcal{B}_{\text {aff }} \rightarrow \mathcal{B}_{W}$ given by $\pi([\alpha, k]):=[\alpha]$.

(2) The algebra $\mathcal{B}_{\text {aff }}$ acts on $S$ via $[\alpha, k] f=\partial_{\alpha}(f)$ for all $k \in \mathbb{Z}$.

Proof. (1) Denote by $\psi$ and $\bar{\psi}$ the braidings on $V_{\text {aff }}$ and $V$ respectively. Let $\tilde{\pi}$ : $\bigoplus_{n} V_{\mathrm{aff}}^{\otimes n} \rightarrow \bigoplus_{n} V^{\otimes n}$ be the lift of $\pi$. Since

$$
\psi([\alpha, k] \otimes[\beta, l])=\left[s_{\alpha}(\beta), l-\left\langle\alpha^{\vee}, \beta\right\rangle k\right] \otimes[\alpha, k]
$$

and $\bar{\psi}([\alpha] \otimes[\beta])=\left[s_{\alpha}(\beta)\right] \otimes[\alpha]$, the map $\tilde{\pi}$ sends the kernel of the braided symmetrizer $\sigma_{n}$ of $V_{\text {aff }}{ }^{\otimes n}$ to that of $V^{\otimes n}$. 
(2) In [2], it is shown that the algebra $\mathcal{B}_{W}$ acts on the coinvariant algebra $S_{W}$ via $[\alpha] \mapsto \partial_{\alpha}$. Let $S^{W}$ be the $W$-invariant subalgebra of $S$. Then we have the decomposition $S=S^{W} \otimes S_{W}$. The operator $\partial_{\alpha}$ extends $S^{W}$-linearly to an operator on $S$. Hence $\mathcal{B}_{W}$ acts on $S$. We have seen the existence of the natural projection $\pi$ from $\mathcal{B}_{\text {aff }}$ to $\mathcal{B}$, so $\pi$ induces the action of $\mathcal{B}_{\text {aff }}$ on $S$.

Let us define the extension $\mathcal{B}_{\text {aff }}(S)=\mathcal{B}_{\text {aff }} \ltimes S$ by the cross relation

$$
[\alpha, k] f=\partial_{\alpha} f+s_{\alpha, 0}(f)[\alpha, k], \quad[\alpha, k] \in V_{\text {aff }}, f \in S .
$$

Proposition 2.1. There exists a homomorphism $\varphi: \mathbb{A} \rightarrow \mathcal{B}_{\text {aff }}(S)$ given by $\tau_{0} \mapsto$ $\left[\alpha_{0},-1\right], \tau_{i} \mapsto\left[\alpha_{i}, 0\right], i=1, \ldots, r$, and $f \mapsto f, f \in S$.

Proof. It is enough to check the Coxeter relations among $\varphi\left(\tau_{0}\right), \ldots, \varphi\left(\tau_{r}\right)$ in $\mathcal{B}_{\text {aff }}(S)$ based on the classification of the affine root systems. This is done by the direct computation of the symmetrizer for the subsystems of rank 2 in a similar manner to $[2$, Section 6$]$.

Example 2.4. We list the Coxeter relations in $\mathcal{B}_{\text {aff }}$ involving $[\theta, 1]=-\left[\alpha_{0},-1\right]$ for the root systems of rank 2. Let $\left(\varepsilon_{1}, \ldots, \varepsilon_{r}\right)$ be an orthonormal basis of the $r$-dimensional Euclidean space. Put $[i j, k]:=\left[\varepsilon_{i}-\varepsilon_{j}, k\right],[\overline{i j}, k]:=\left[\varepsilon_{i}+\varepsilon_{j}, k\right]$, $[i, k]:=\left[\varepsilon_{i}, k\right]$ and $[\alpha]:=[\alpha, 0]$.

(i) (Type $A_{2}$ case)

$$
[13,1][23][13,1]+[23][13,1][23]=0, \quad[13,1][12][13,1]+[12][13,1][12]=0 .
$$

(ii) (Type $B_{2}$ case)

$$
[\overline{12}, 1][2][\overline{12}, 1][2]=[2][\overline{12}, 1][2][\overline{12}, 1] .
$$

(iii) (Type $G_{2}$ case) Let $\alpha_{1}, \alpha_{2}$ be the simple roots for the $G_{2}$-system. We assume that $\alpha_{1}$ is a short root and $\alpha_{2}$ is a long one. Then we have $\theta=3 \alpha_{1}+2 \alpha_{2}$, and

$$
[\theta, 1]\left[\alpha_{2}\right][\theta, 1]+\left[\alpha_{2}\right][\theta, 1]\left[\alpha_{2}\right]=0
$$

\section{$\S 3$. Model of nil-Hecke algebra}

The connected components of $P \otimes \mathbb{R} \backslash \bigcup_{\alpha \in \Delta_{+}, k \in \mathbb{Z}} H_{\alpha, k}$ are called alcoves. The affine Weyl group $W_{\text {aff }}$ acts on the set of alcoves simply transitively.

Definition 3.1 ([8]). (1) A sequence $\left(A_{0}, \ldots, A_{l}\right)$ of alcoves $A_{i}$ is called an alcove path if $A_{i}$ and $A_{i+1}$ have a common wall and $A_{i} \neq A_{i+1}$.

(2) An alcove path $\left(A_{0}, \ldots, A_{l}\right)$ is called reduced if the length $l$ of the path is minimal among all alcove paths connecting $A_{0}$ and $A_{l}$. 
(3) We use the symbol $A_{i} \stackrel{\beta, k}{\longrightarrow} A_{i+1}$ when $A_{i}$ and $A_{i+1}$ have a common wall of the form $H_{\beta, k}$ and the direction of the $\operatorname{root} \beta$ is from $A_{i}$ to $A_{i+1}$.

The alcove $A^{\circ}$ defined by the inequalities $\left\langle\lambda, \alpha_{0}\right\rangle \geq-1$ and $\left\langle\lambda, \alpha_{i}\right\rangle \geq 0$, $i=1, \ldots, r$, is called the fundamental alcove. For a reduced alcove path $\gamma: A_{0}=$ $A^{\circ} \stackrel{\beta_{1}, k_{1}}{\longrightarrow} \cdots \stackrel{\beta_{l}, k_{l}}{\longrightarrow} A_{l}$, we define an element $[\gamma] \in \mathcal{B}_{\text {aff }}$ by

$$
[\gamma]:=\left[-\beta_{1},-k_{1}\right] \cdots\left[-\beta_{l},-k_{l}\right] .
$$

When $A_{l}=x^{-1}\left(A^{\circ}\right)$ for $x \in W_{\text {aff }}$, we will also use the symbol $[x]$ instead of $[\gamma]$, since $[\gamma]$ depends only on $x$ thanks to the Yang-Baxter relations listed in Example 2.4 .

For a braided vector space $M$, it is known that an element $a \in M$ acts on $\mathcal{B}\left(M^{*}\right)$ as a braided differential operator (see [2], [9], [11]). Let us identify $M^{*}$ with $M$ via the $W_{\text {aff-invariant inner product }}($, ) given by

$$
([\alpha, k],[\beta, l])= \begin{cases}1 & \text { if } \alpha=\beta \text { and } k=l, \\ 0 & \text { otherwise }\end{cases}
$$

for $\alpha, \beta \in \Delta_{+}$and $k, l \in \mathbb{Z}$. In our case, the differential operator $\overleftarrow{D}_{[\alpha, k]},[\alpha, k] \in$ $V_{\text {aff }}$, acting from the right is determined by the following conditions:

(0) $(c) \overleftarrow{D}_{[\alpha, k]}=0$ for $c \in \mathbb{Q}$

(1) $([\alpha, k]) \overleftarrow{D}_{[\beta, l]}=([\alpha, k],[\beta, l])$

(2) $(F G) \overleftarrow{D}_{[\alpha, k]}=F\left(G \overleftarrow{D}_{[\alpha, k]}\right)+\left(F \overleftarrow{D}_{[\alpha, k]}\right) s_{\alpha, k}(G)$

for $\alpha, \beta \in \Delta, k, l \in \mathbb{Z}$ and $F, G \in \mathcal{B}_{\text {aff }}$. The operator $\overleftarrow{D}_{[\alpha, k]}$ extends to one acting on $\mathcal{B}_{\text {aff }}(S)$ by the commutation relation $f \cdot \overleftarrow{D}_{[\alpha, k]}=\overleftarrow{D}_{[\alpha, k]} \cdot s_{\alpha, k}(f), f \in S$

We use the abbreviations $\overleftarrow{D}_{0}:=\overleftarrow{D}_{\left[\alpha_{0},-1\right]}$ and $\overleftarrow{D}_{i}:=\overleftarrow{D}_{\left[\alpha_{i}, 0\right]}, i=1, \ldots, r$. For $x \in W_{\text {aff }}$, fix a reduced decomposition $x=s_{i_{1}} \cdots s_{i_{l}}$. We define the corresponding braided differential operator $\overleftarrow{D}_{x}$ acting on $\mathcal{B}_{\text {aff }}$ by the formula

$$
\overleftarrow{D}_{x}:=\overleftarrow{D}_{i_{l}} \ldots \overleftarrow{D}_{i_{1}}
$$

which is also independent of the choice of the reduced decomposition of $x$ because of the braid relations.

Lemma 3.2. For $x \in W_{\text {aff }}$, take a reduced alcove path $\gamma$ from the fundamental alcove $A^{\circ}$ to $x^{-1}\left(A^{\circ}\right)$. Then $([\gamma]) \overleftarrow{D}_{x}=1$

Proof. Take a reduced path

$$
\gamma: A_{0}=A^{\circ} \stackrel{\beta_{1}, k_{1}}{\longrightarrow} A_{1} \stackrel{\beta_{2}, k_{2}}{\longrightarrow} \cdots \stackrel{\beta_{l}, k_{l}}{\longrightarrow} A_{l}=x^{-1}\left(A^{\circ}\right) .
$$


Define a sequence $\sigma_{1}, \ldots, \sigma_{l} \in W_{\text {aff }}$ inductively by

$$
\sigma_{1}:=s_{\beta_{1}, k_{1}}, \quad \sigma_{j+1}:=\sigma_{j} \sigma_{j-1} \cdots \sigma_{1} \cdot s_{\beta_{j+1}, k_{j+1}} \cdot \sigma_{1} \cdots \sigma_{j-1} \sigma_{j} .
$$

Then it is easy to see that $\sigma_{\nu} \cdots \sigma_{1}\left(A_{j}\right) \neq A^{\circ}, 1 \leq \nu \leq j-1, \sigma_{j} \cdots \sigma_{1}\left(A_{j}\right)=A^{\circ}$ and the walls $\sigma_{j} \cdots \sigma_{1}\left(H_{\beta_{j+1}, k_{j+1}}\right)$ correspond to simple roots. Hence, $\sigma_{1}, \ldots, \sigma_{l}$ are simple reflections. This sequence gives a reduced expression $x=\sigma_{l} \cdots \sigma_{1}$. Put $\sigma_{i}=s_{\alpha_{i_{j}}}$. Since the direction of $\beta_{j+1}$ is chosen to be from $A_{j}$ to $A_{j+1}$, we have

$$
[\gamma] \overleftarrow{D}_{x}=\left(\left[\beta_{1}, k_{1}\right]\right) \overleftarrow{D}_{i_{1}} \cdot\left(\sigma_{1}\left(\left[\beta_{2}, k_{2}\right]\right)\right) \overleftarrow{D}_{i_{2}} \cdots\left(\sigma_{l-1} \cdots \sigma_{1}\left(\left[\beta_{l}, k_{l}\right]\right)\right) \overleftarrow{D}_{i_{l}}=1
$$

Example $3.3\left(A_{2}\right.$-case). The standard realization is given by $\alpha_{1}=\varepsilon_{1}-\varepsilon_{2}, \alpha_{2}=$ $\varepsilon_{2}-\varepsilon_{3}, \alpha_{0}=\varepsilon_{3}-\varepsilon_{1}$. Consider the translation $t_{\alpha_{1}}$ by the simple root $\alpha_{1}$. If we take a reduced path

$$
\gamma: A_{0}=A^{\circ} \stackrel{-\alpha_{2}, 0}{\longrightarrow} A_{1} \stackrel{\alpha_{1}, 1}{\longrightarrow} A_{2} \stackrel{-\alpha_{0}, 1}{\longrightarrow} A_{3} \stackrel{\alpha_{1}, 2}{\longrightarrow} A_{4}=t_{\alpha_{1}}\left(A^{\circ}\right),
$$

then we have $[\gamma]=[23][21,-1][31,-1][21,-2]$. On the other hand, the differential operator corresponding to $t_{-\alpha_{1}}$ is given by $\overleftarrow{D}_{2} \overleftarrow{D}_{0} \overleftarrow{D}_{2} \overleftarrow{D}_{1}$, where $\overleftarrow{D}_{0}=$ $\overleftarrow{D}_{[31,-1]}, \overleftarrow{D}_{1}=\overleftarrow{D}_{[12]}, \overleftarrow{D}_{2}=\overleftarrow{D}_{[23]}$. It is easy to check by direct computation that

$$
([23][21,-1][31,-1][12,2]) \overleftarrow{D}_{2} \overleftarrow{D}_{0} \overleftarrow{D}_{2} \overleftarrow{D}_{1}=1
$$

Theorem 3.4. The algebra homomorphism $\varphi: \mathbb{A} \rightarrow \mathcal{B}_{\text {aff }}(S)$ is injective.

Proof. The nil-Hecke algebra $\mathbb{A}$ is also $W_{\text {aff-graded by assigning the } W_{\text {aff-degree }}}$ to each generator by $\operatorname{deg}_{W_{\text {aff }}}\left(\tau_{i}\right)=s_{i}$. Since the homomorphism $\varphi: \mathbb{A} \rightarrow \mathcal{B}_{\text {aff }}(S)$

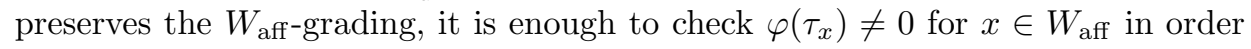
to show the injectivity of $\varphi$. On the other hand, $\mathcal{B}_{\text {aff }}^{\text {op }}$ acts on $\mathcal{B}_{\text {aff }}$ itself via the braided differential operators. Let $\gamma$ be a reduced alcove path from $A^{\circ}$ to $x^{-1}\left(A^{\circ}\right)$. Then $([\gamma]) \overleftarrow{D}_{x}=1$ from Lemma 3.2 . This shows $\overleftarrow{D}_{x} \neq 0$, so $\varphi\left(\tau_{x}\right) \neq 0$

This theorem implies the following (see Proposition 1.1):

Corollary 3.5. The T-equivariant Pontryagin ring $H_{*}^{T}(\widehat{\mathrm{Gr}})$ is a subalgebra of $\mathcal{B}_{\text {aff }}(S)$.

By taking the non-equivariant limit, we also have:

Corollary 3.6. The Pontryagin ring $H_{*}(\widehat{\mathrm{Gr}})$ is a subalgebra of $\mathcal{B}_{\mathrm{aff}}$.

\section{§4. Affine Bruhat operators}

We denote by $x \rightarrow y$ the cover relation in the Bruhat ordering of $W_{\text {aff }}$, i.e. $y=x s_{\alpha, k}$ for some $\alpha \in \Delta$ and $k \in \mathbb{Z}$, and $l(y)=l(x)+1$. 
We will use some terminology from [7]. Denote by $\tilde{Q}$ the set of antidominant elements in $Q^{\vee}$. An element $x \in W_{\text {aff }}$ can be uniquely expressed as a product of the form $x=w t_{v \lambda} \in W_{\text {aff }}$ with $v, w \in W, \lambda \in \tilde{Q}$. We say that $x=w t_{v \lambda}$ belongs to the $v$-chamber. An element $\lambda \in \tilde{Q}$ is called superregular when $|\langle\lambda, \alpha\rangle|>2(\# W)+2$ for all $\alpha \in \Delta_{+}$. If $\lambda \in \tilde{Q}$ is superregular, then $x=w t_{v \lambda}$ is also called superregular. The subset of superregular elements in $W_{\text {aff }}$ is denoted by $W_{\text {aff }}^{\text {sreg }}$. We say that a property holds for sufficiently superregular elements $W_{\text {aff }}^{\text {ssreg }} \subset W_{\text {aff }}$ if there is a positive constant $k \in \mathbb{Z}$ such that the property holds for all $x \in W_{\text {aff }}^{\text {sreg }}$ satisfying the following condition:

$$
y \in W_{\text {aff }}, y<x \text {, and } l(x)-l(y)<k \Rightarrow y \in W_{\text {aff }^{\text {sreg }}} \text {. }
$$

The meaning of $W_{\text {aff }}^{\text {ssreg }}$ depends on the context (see [7, Section 4] for the details). For $v \in W$, consider the $S$-submodule $M_{v}^{\text {ssreg }}$ in $\mathcal{B}_{\text {aff }}$ generated by the sufficiently superregular elements $[x]$ where $x$ belongs to the $v$-chamber.

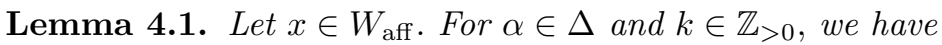

$$
[x] \overleftarrow{D}_{[\alpha, k]}= \begin{cases}{\left[x s_{\alpha, k}\right]} & \text { if } l(x)=l\left(x s_{\alpha, k}\right)+1 \\ 0 & \text { otherwise }\end{cases}
$$

Proof. The fundamental alcove $A^{\circ}$ is contained in the region $\{\lambda \in P \otimes \mathbb{R} \mid\langle\lambda, \alpha\rangle$ $<k\}$ for $\alpha \in \Delta$ and $k \in \mathbb{Z}_{>0}$. Choose any reduced path $\gamma: A_{0} \stackrel{\beta_{1}, k_{1}}{\longrightarrow} \cdots \stackrel{\beta_{l}, k_{l}}{\longrightarrow}$ $A_{l}=x^{-1}\left(A^{\circ}\right)$ with $k_{i} \geq 0$. If $l(x)>l\left(x s_{\alpha, k}\right)$, then $\left(\beta_{i}, k_{i}\right)=(\alpha, k)$ for some $i$. Take the largest $i$ and consider the path

$$
\begin{array}{r}
\gamma^{\prime}: A_{0} \stackrel{\beta_{1}, k_{1}}{\longrightarrow} \cdots \stackrel{\beta_{i-1}, k_{i-1}}{\longrightarrow} A_{i-1} \stackrel{\beta_{i+1}^{\prime}, k_{i+1}^{\prime}}{\longrightarrow} s_{\alpha, k}\left(A_{i+1}\right) \stackrel{\beta_{i+2}^{\prime}, k_{i+2}^{\prime}}{\longrightarrow} \cdots \\
\cdots \stackrel{\beta_{l}^{\prime}, k_{l}^{\prime}}{\longrightarrow} s_{\alpha, k}\left(A_{l}\right)=s_{\alpha, k} x^{-1}\left(A^{\circ}\right)=\left(x s_{\alpha, k}\right)^{-1}\left(A^{\circ}\right),
\end{array}
$$

where $\left(\beta_{j}^{\prime}, k_{j}^{\prime}\right)$ is determined by the condition $s_{\alpha, k}\left(H_{\beta_{j}, k_{j}}\right)=H_{\beta_{j}^{\prime}, k_{j}^{\prime}}$. If $l(x)=$ $l\left(x s_{\alpha, k}\right)+1$, then $\gamma^{\prime}$ is a reduced path. In this case, $[x] \overleftarrow{D}_{[\alpha, k]}=\left[x s_{\alpha, k}\right]$. If $l(x)>$ $l\left(x s_{\alpha, k}\right)+1$, then $\gamma^{\prime}$ is not reduced and $[x] \overleftarrow{D}_{[\alpha, k]}=0$. When $l(x)<l\left(x s_{\alpha, k}\right)$, the element $[\alpha, k]$ does not appear in the monomial $[\gamma]$, so $[x] \overleftarrow{D}_{[\alpha, k]}=0$

Proposition 4.1 ([7, Proposition 4.1]). Let $\lambda \in \tilde{Q}$ be superregular. For $x=w t_{v \lambda}$ and $y=x s_{v \alpha,-n}$ with $v, w \in W$, we have the cover relation $y \rightarrow x$ if and only if one of the following conditions holds:

(1) $l(w v)=l\left(w v s_{\alpha}\right)-1$ and $n=\langle\lambda, \alpha\rangle$, giving $y=w s_{v(\alpha)} t_{v(\lambda)}$,

(2) $l(w v)=l\left(w v s_{\alpha}\right)+\left\langle\alpha^{\vee}, 2 \rho\right\rangle-1$ and $n=\langle\lambda, \alpha\rangle+1$, giving $y=w s_{v(\alpha)} t_{v\left(\lambda+\alpha^{\vee}\right)}$, 
(3) $l(v)=l\left(v s_{\alpha}\right)+1$ and $n=0$, giving $y=w s_{v(\alpha)} t_{v s_{\alpha}(\lambda)}$,

(4) $l(v)=l\left(v s_{\alpha}\right)-\left\langle\alpha^{\vee}, 2 \rho\right\rangle+1$ and $n=-1$, giving $y=w s_{v(\alpha)} t_{v s_{\alpha}\left(\lambda+\alpha^{\vee}\right)}$.

In [7], the first parts of conditions (1) and (2) are called the near relation because $x$ and $y$ belong to the same chamber. In this paper we denote the near relation by $y \rightarrow_{\text {near }} x$.

The affine Bruhat operator $B^{\mu}: S\left\langle W_{\mathrm{aff}}{ }^{\text {ssreg }}\right\rangle \rightarrow S\left\langle W_{\mathrm{aff}}{ }^{\text {sreg }}\right\rangle, \mu \in P$, due to Lam and Shimozono [7, Section 5] is an $S$-linear map defined by the formula

$$
B^{\mu}(x)=(\mu-w v \mu) x+\sum_{\alpha \in \Delta_{+}} \sum_{x s_{v(\alpha), k} \rightarrow \text { near } x}\left\langle\alpha^{\vee}, \mu\right\rangle x s_{v(\alpha), k}
$$

for $x=w t_{v \lambda} \in W_{\text {aff }}^{\text {ssreg }}$. We also introduce the operator $\beta_{v}^{\mu}, \mu \in P$, acting on each $M_{v}^{\text {ssreg }}$ by

$$
\beta_{v}^{\mu}([x]):=(\mu-w v \mu)[x]+[x] \sum_{\alpha \in \Delta_{+}, k>1}\left\langle\alpha^{\vee}, \mu\right\rangle \overleftarrow{D}_{[v(\alpha), k]}
$$

where $x=w t_{v \lambda} \in W_{\mathrm{aff}}^{\text {ssreg }}$. Denote by $W_{\text {aff }}^{\text {ssreg }}(v)$ the subset of $W_{\text {aff }}$ consisting of the superregular elements belonging to the $v$-chamber. Fix a left $S$-module isomorphism

$$
\iota: S\left\langle W_{\mathrm{aff}}^{\mathrm{ssreg}}(v)\right\rangle \rightarrow M_{v}^{\mathrm{ssreg}}, \quad x \mapsto[x] .
$$

Proposition 4.2. For each $v \in W$ and a sufficiently superregular element $x \in$ $W_{\text {aff }}^{\text {ssreg }}(v)$,

$$
\beta_{v}^{\mu}([x])=\iota\left(B^{\mu}(x)\right) .
$$

Proof. This can be shown by using Lemma 4.1 and Proposition 4.1:

$$
\begin{aligned}
\beta_{v}^{\mu}([x]) & =(\mu-w v \mu)[x]+[x] \sum_{\alpha \in \Delta_{+}, k>1}\left\langle\alpha^{\vee}, \mu\right\rangle \overleftarrow{D}_{[v(\alpha), k]} \\
& =(\mu-w v \mu)[x]+\sum_{\alpha \in \Delta_{+}} \sum_{k>1, l\left(x s_{v(\alpha), k}\right)=l(x)-1}\left\langle\alpha^{\vee}, \mu\right\rangle\left[x s_{v(\alpha), k}\right] \\
& =(\mu-w v \mu)[x]+\sum_{\alpha \in \Delta_{+}} \sum_{x s_{v(\alpha), k} \rightarrow_{\text {near }} x}\left\langle\alpha^{\vee}, \mu\right\rangle\left[x s_{v(\alpha), k}\right]=\iota\left(B^{\mu}(x)\right)
\end{aligned}
$$

Remark 4.2. In [5] the authors introduced the quantization operators $\eta_{\alpha}$ acting on the model of $H^{*}(G / B) \otimes \mathbb{C}\left[q_{1}, \ldots, q_{r}\right]$ realized as a subalgebra of $\mathcal{B}_{W} \otimes$ $\mathbb{C}\left[q_{1}, \ldots, q_{r}\right]$. For a superregular element $\lambda \in \tilde{Q}$ and $w \in W$, consider a homomorphism $\theta_{w}^{\lambda}$ from the $\lambda$-small elements (see $\left[7\right.$, Section 5]) of $H^{*}(G / B) \otimes \mathbb{C}[q]$ to $\mathcal{B}_{\text {aff }}$ defined by

$$
\theta_{w}^{\lambda}\left(q^{\mu} \sigma^{v}\right):=\left[v w^{-1} t_{w(\lambda+\mu)}\right],
$$


where $\sigma^{v}$ is the Schubert class of $G / B$ corresponding to $v \in W$ and $q^{\mu}=q_{1}^{\mu_{1}} \cdots q_{r}^{\mu_{r}}$ for $\mu=\sum_{i=1}^{r} \mu_{i} \alpha_{i}^{\vee}$. The following is an interpretation of the formula of [7, Proposition 5.1] in our setting:

$$
\theta_{w}^{\lambda}\left(\eta_{\alpha}(\sigma)\right)=\beta_{w}^{\varpi \alpha}\left(\theta_{w}^{\lambda}(\sigma)\right) .
$$

\section{$\S 5$. Peterson isomorphism}

As seen in the proof of Lemma 3.1, we can read off two kinds of decomposition of an element $x \in W_{\text {aff }}$ into products of reflections from an alcove path

$$
\gamma: A_{0}=A^{\circ} \stackrel{\beta_{1}, k_{1}}{\longrightarrow} A_{1} \stackrel{\beta_{2}, k_{2}}{\longrightarrow} \cdots \stackrel{\beta_{l}, k_{l}}{\longrightarrow} A_{l}=x^{-1}\left(A^{\circ}\right) .
$$

One of them is a reduced decomposition $x=\sigma_{l} \cdots \sigma_{1}$, and another is a decomposition $x=s_{\beta_{l}, k_{l}} \cdots s_{\beta_{1}, k_{1}}$ into a product of (not necessarily simple) reflections. Let $M$ be a left $S$-submodule of $\mathcal{B}_{\text {aff }}(S)$ generated by the elements $[x], x \in W_{\text {aff }}$. The correspondence between the above decompositions of $x$ induces an isomorphism of $S$-modules:

$$
v: M \rightarrow \varphi(\mathbb{A}) \cong \mathbb{A}, \quad[x]=\left[-\beta_{1},-k_{1}\right] \cdots\left[-\beta_{l},-k_{l}\right] \mapsto \varphi\left(\tau_{x}\right)=\left[\sigma_{l}\right] \cdots\left[\sigma_{1}\right] .
$$

For a superregular antidominant element $\lambda \in Q$ and $\mu_{1}, \ldots, \mu_{k} \in P$, we see that $v\left(\sum_{w \in W} \beta_{w}^{\mu_{k}} \cdots \beta_{w}^{\mu_{1}}\left[t_{w \lambda}\right]\right)$ belongs to $Z_{\mathbb{A}}(S)$ from [7, Theorem 7.2].

The Peterson isomorphism

$$
\begin{aligned}
\psi: H_{*}^{T}(\widehat{\mathrm{Gr}})_{\mathrm{loc}}:=H_{*}^{T}(\widehat{\mathrm{Gr}})\left[\xi_{t_{\lambda}}^{-1} \mid \lambda \in \tilde{Q}\right] & \\
& \rightarrow Q H_{T}^{*}(G / B)_{\mathrm{loc}}:=Q H^{*}(G / B)\left[q_{1}^{-1}, \ldots, q_{r}^{-1}\right]
\end{aligned}
$$

is an $S$-algebra isomorphism given by

$$
\xi_{w t_{\lambda}} \xi_{t_{\mu}} \mapsto q^{\lambda-\mu} \sigma^{w}, \quad w \in W, \lambda, \mu \in \tilde{Q} .
$$

Proposition 5.1. The Peterson isomorphism $\psi$ is characterized by the condition

$$
\psi\left(j^{-1}\left(v\left(\sum_{w \in W} \beta_{w}^{\mu_{k}} \cdots \beta_{w}^{\mu_{1}}\left[t_{w \lambda}\right]\right)\right)\right)=q^{\lambda} \eta_{\mu_{1}} \cdots \eta_{\mu_{k}} \sigma^{\mathrm{id}}
$$

for a superregular element $\lambda \in \tilde{Q}$ and $\mu_{1}, \ldots \mu_{k} \in P$.

\section{$\S 6$. Quadratic relations}

For $\alpha \in \Delta_{+}$and $v \in W$, define the operator $\mathbb{D}_{v}(\alpha)$ by

$$
\mathbb{D}_{v}(\alpha):=\sum_{k>1} \overleftarrow{D}_{[v(\alpha), k]}
$$


Then

$$
\beta_{v}^{\mu}([x])=(\mu-w v \mu)[x]+[x] \sum_{\alpha \in \Delta_{+}}\left\langle\alpha^{\vee}, \mu\right\rangle \mathbb{D}_{v}(\alpha) .
$$

In the following, we discuss the relations among the operators $\mathbb{D}_{v}(\alpha), \alpha \in \Delta_{+}$, for a root system of type $A_{n-1}$. For simplicity, we consider only the non-equivariant case with $v=\mathrm{id}$. Take the standard realization of the $A_{n-1}$-system:

$$
\Delta=\left\{\varepsilon_{i}-\varepsilon_{j} \mid 1 \leq i, j \leq n, i \neq j\right\} .
$$

Put $\mathbb{D}(i j):=\mathbb{D}_{\text {id }}\left(\varepsilon_{i}-\varepsilon_{j}\right)$ for $1 \leq i<j \leq n$, and $\mathbb{D}(i j):=-\mathbb{D}(j i)$ for $i>j$. In this situation, we have a formula for the non-equivariant limit $\bar{\beta}_{\text {id }}^{\varepsilon_{i}}$ of the operator $\beta_{\text {id }}^{\varepsilon_{i}}$ :

$$
\bar{\beta}_{\mathrm{id}}^{\varepsilon_{i}}=\sum_{j \neq i} \mathbb{D}(i j)
$$

Note that this formula is analogous to the definition of the Dunkl elements in [4].

Let $T_{i}, 1 \leq i \leq n-1$, be linear operators on $M^{\text {ssreg }}$ defined by $T_{i}([x]):=\left[x t_{\alpha_{i}}\right]$, where $x \in W_{\text {aff }}$ and $\alpha_{i}=\varepsilon_{i}-\varepsilon_{i+1}$. It is easy to check from Proposition 4.1 that $\left(T_{i}[x]\right) \mathbb{D}(j k)=T_{i}([x] \mathbb{D}(j k))$. Our next goal is to show that the operators $\mathbb{D}(i j)$ satisfy the defining relations of the quantum deformation $\mathcal{E}_{n}^{q}$ of the Fomin-Kirillov quadratic algebra [4].

Proposition 6.1. (i) For $1 \leq i<j \leq n$, we have

$$
\mathbb{D}(i j)^{2}= \begin{cases}T_{i} & \text { if } j=i+1, \\ 0 & \text { otherwise. }\end{cases}
$$

(ii) If $\{i, j\} \cap\{k, l\}=\emptyset$, then $\mathbb{D}(i j) \mathbb{D}(k l)=\mathbb{D}(k l) \mathbb{D}(i j)$.

(iii) For $1 \leq i, j \leq n, i \neq j$,

$$
\mathbb{D}(i j) \mathbb{D}(j k)+\mathbb{D}(j k) \mathbb{D}(k l)+\mathbb{D}(k i) \mathbb{D}(i j)=0 .
$$

Proof. First of all, let us check (i). We have

$$
\mathbb{D}(i j)^{2}=\sum_{k, l>1} \overleftarrow{D}_{[i j, k]} \overleftarrow{D}_{[i j, l]}
$$

Let $\lambda \in \tilde{Q}$ be sufficiently superregular. For $x=w t_{\lambda} \in W_{\text {aff }}$, assume that

$$
[x] \overleftarrow{D}_{[i j, k]} \overleftarrow{D}_{[i j, l]} \neq 0
$$

Then we have the arrows $x s_{i j, k} \rightarrow_{\text {near }} x$ and $x s_{i j, k} s_{i j, l} \rightarrow_{\text {near }} x s_{i j, k}$ in the Bruhat ordering. From conditions (1) and (2) of Proposition 4.1, one of the following conditions holds: 
Case 1: $k=-\left\langle\lambda, \varepsilon_{i}-\varepsilon_{j}\right\rangle$ and $l(w)=l\left(w s_{i j}\right)-1$.

Case 2: $k=-\left\langle\lambda, \varepsilon_{i}-\varepsilon_{j}\right\rangle-1$ and $l(w)=l\left(w s_{i j}\right)+\left\langle\varepsilon_{i}-\varepsilon_{j}, 2 \rho\right\rangle-1$.

In Case 1 , since the arrow $x s_{i j, k} s_{i j, l}=w s_{i j} t_{\lambda} s_{i j, l} \rightarrow_{\text {near }} x s_{i j, k}$ must come from condition (2) of Proposition 4.1, we have $\left\langle\varepsilon_{i}-\varepsilon_{j}, 2 \rho\right\rangle-1=1$. This equality implies that $\varepsilon_{i}-\varepsilon_{j}$ is a simple root $\alpha_{i}$, and we get

$$
[x] \mathbb{D}(i i+1)^{2}=[x] \overleftarrow{D}_{\left[\alpha_{i},-\left\langle\lambda, \alpha_{i}\right\rangle\right]} \overleftarrow{D}_{\left[\alpha_{i},-\left\langle\lambda, \alpha_{i}\right\rangle-1\right]}=\left[x t_{\alpha_{i}}\right]=T_{i}[x]
$$

In Case 2, since the arrow $x s_{i j, k} s_{i j, l}=w s_{i j} t_{\lambda+\varepsilon_{i}-\varepsilon_{j}} s_{i j, l} \rightarrow_{\text {near }} x s_{i j, k}$ comes from condition (1) of Proposition 4.1, we again obtain $\left\langle\varepsilon_{i}-\varepsilon_{j}, 2 \rho\right\rangle-1=1$ and $\varepsilon_{i}-\varepsilon_{j}$ $=\alpha_{i}$. Hence we get

$$
[x] \mathbb{D}(i i+1)^{2}=[x] \overleftarrow{D}_{\left[\alpha_{i},-\left\langle\lambda, \alpha_{i}\right\rangle-1\right]} \overleftarrow{D}_{\left[\alpha_{i},-\left\langle\lambda, \alpha_{i}\right\rangle-2\right]}=\left[x t_{\alpha_{i}}\right]=T_{i}[x]
$$

If $j \neq i+1$, we have $\mathbb{D}(i j)^{2}=0$. Relations (ii) and (iii) follow from the identities $[i j, a][k l, b]=[k l, b][i j, a]$ for $\{i, j\} \cap\{k, l\}=\emptyset$, and

$$
[i j, a][j k, b]+[j k, b][k i,-a-b]+[k i,-a-b][i j, a]=0
$$

in $\mathcal{B}_{\text {aff }}$.

Remark 6.1. The operators $\mathbb{D}_{v}(\alpha)$ induce the quantum Bruhat representation of $\mathcal{E}_{n}^{q}$ via $\theta_{v}^{\lambda}$.

\section{Acknowledgements}

The second author is supported by Grant-in-Aid for Scientific Research.

\section{References}

[1] N. Andruskiewitsch and H.-J. Schneider, Finite quantum groups and Cartan matrices, Adv. Math. 154 (2000), 1-45. Zbl 1007.16027 MR 1780094

[2] Y. Bazlov, Nichols-Woronowicz algebra model for Schubert calculus on Coxeter groups, J. Algebra 297 (2006), 372-399. Zbl 1101.16027 MR 2209265

[3] R. Bott, The space of loops on a Lie group, Michigan Math. J. 5 (1958), 35-61. Zbl 0096.17701 MR 0102803

[4] S. Fomin and A. N. Kirillov, Quadratic algebras, Dunkl elements and Schubert calculus, in Advances in geometry, J.-L. Brylinski et al. (eds.), Progr. Math. 172, Birkhäuser, 1995, 147-182. Zbl 0940.05070 MR 1667680

[5] A. N. Kirillov and T. Maeno, A note on quantization operators on Nichols algebra model for Schubert calculus on Weyl groups, Lett. Math. Phys. 72 (2005), 233-241. Zbl 1104.14021 MR 2164613

[6] B. Kostant and S. Kumar, The nil Hecke ring and cohomology of $G / P$ for a Kac-Moody group $G$, Adv. Math. 62 (1986), 187-237. Zbl 0641.17008 MR 0866159

[7] T. Lam and M. Shimozono, Quantum cohomology of $G / P$ and homology of affine Grassmannian, Acta Math. 24 (2010), 49-90. Zbl 1216.14052 MR 2600433 
[8] C. Lenart and A. Postnikov, Affine Weyl groups in $K$-theory and representation theory, Int. Math. Res. Notices 2007, no. 12, art. ID rnm038, 65 pp. Zbl 1137.14037 MR 2344548

[9] S. Majid, Free braided differential calculus, braided binomial theorem, and the braided exponential map, J. Math. Phys. 34 (1993), 4843-4856. Zbl 0807.16035 MR 1235979

[10] Double bosonisation of braided groups and the construction of $U_{q}(\mathfrak{g})$, Math. Proc. Cambridge Philos. Soc. 125 (1999), 151-192. Zbl 0974.17018 MR 1645545

[11] _ Noncommutative differentials and Yang-Mills on permutation groups $S_{N}$, in Hopf algebras in noncommutative geometry and physics, Lecture Notes in Pure Appl. Math. 239, Dekker, 2004, 189-213. Zbl 1076.58004 MR 2106930

[12] A. Milinski and H.-J. Schneider, Pointed indecomposable Hopf algebras over Coxeter groups, in New trends in Hopf algebra theory (La Falda, 1999), Contemp. Math. 267, Amer. Math. Soc., 2000, 215-236. Zbl 1093.16504 MR 1800714

[13] W. D. Nichols, Bialgebras of type one, Comm. Algebra 6 (1978), 1521-1552. Zbl 0408.16007 MR 0506406

[14] D. Peterson, Lecture notes at MIT, 1997.

[15] S. L. Woronowicz, Differential calculus on compact matrix pseudogroups (quantum groups), Comm. Math. Phys. 122 (1989), 125-170. Zbl 0751.58042 MR 0994499 